\title{
Successful Removal of Metal Rings Causing Penile Strangulation
}

\author{
Stephanie Purnell ${ }^{1}$, Patrick Lam ${ }^{1}$, Christina Lipscombe ${ }^{2}$, Dynell Pinder ${ }^{1}$, \\ Dr.Chiledum Ahaghotu ${ }^{1}$, Dr.Pamela Coleman ${ }^{1}$ \\ ${ }^{1}$ Division of Urology, Howard University Hospital, Washington, DC, USA \\ ${ }^{2}$ Philadelphia College of Osteopathic Medicine, Philadelphia, PA, USA
}

\begin{abstract}
While not a new phenomenon, genital incarceration or strangulation is an infrequent urological emergency. Incarceration occurs due to constriction of the genitals by a device such as a metal ring, rubber ring, or rubber band. Localized edema, superficial ulcers, and skin loss are the most common complications of strangulation, but with prompt attention to the injury, patients can have an excellent prognosis. While the technique used to remove the constriction device varies, the urologist should act swiftly but prudently to decompress the genitals. Our case report discusses the epidemiology and anatomy of genital incarceration by two metal cock rings, as well as a novel and safe approach, using the Midas Rex orthopedic drill, to remove the metal rings from the base of the patient's penis and scrotum.
\end{abstract}

Keywords - Genital incarceration, urological emergency, constriction device, decompression, how-to-guide

\section{INTRODUCTION}

Although not a new phenomenon, genital strangulation or incarceration is an infrequent urological emergency (1). Localized edema, superficial ulcers, and skin loss are the most common complications of strangulation, but with prompt attention to the injury, patients can leave in excellent condition (2). Without prompt attention and intervention, the patient is susceptible to vascular occlusion, ischemia, necrosis, erectile dysfunction, urethral-cutaneous fistula, and ultimately penile amputation which is an unusual but devastating consequence (3). The urologist should act swiftly but prudently to decompress the genitals, and the technique used depends on the constriction device used (metal ring, rubber ring, or rubber band (4)(5). We describe below a case of strangulation and the surgical technique used to remove the metal constriction device.

\section{CASE PRESENTATION}

A 33 year old African American male presented to the emergency department (ED) complaining of worsening penile and scrotal pain, and swelling after placing two metal cock rings around his genitals. The patient stated that he used the rings to enhance pleasure and to prolong his erection during sexual intercourse. He attempted to remove the rings with both water and oil based lubricants but he was unsuccessful, and 8 hours later, he arrived at the ED. The patient was in obvious discomfort and was concerned that the swelling was worsening. On physical examination, the penis and scrotum were incarcerated by two metal rings located at the base of the organs (Fig. 1). The skin underneath the rings was still intact, but there was significant swelling of the proximal penis and the scrotum (Fig. 2). The affected area was exquisitely tender to palpation. Urgent urologic consultation was requested. Attempting to mechanically excise the rings with bolt cutters was not plausible and no other methods of removal in the ED were sound.

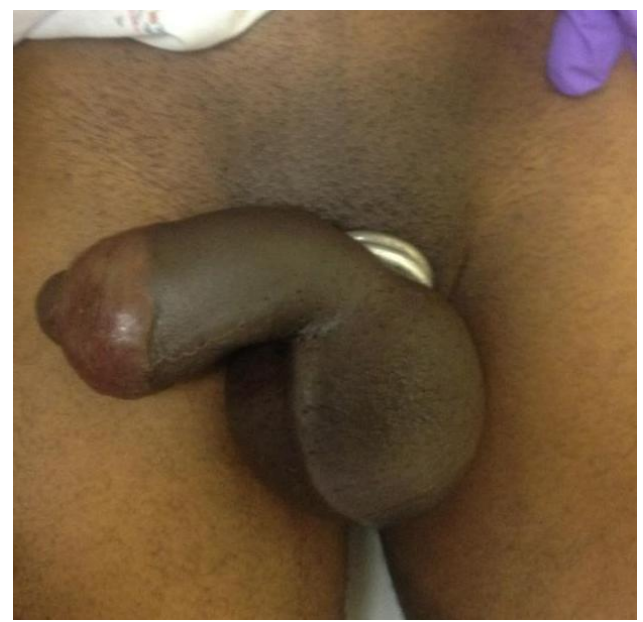

Figure 1: Frontal view demonstrating incarceration of the penis and scrotum. 


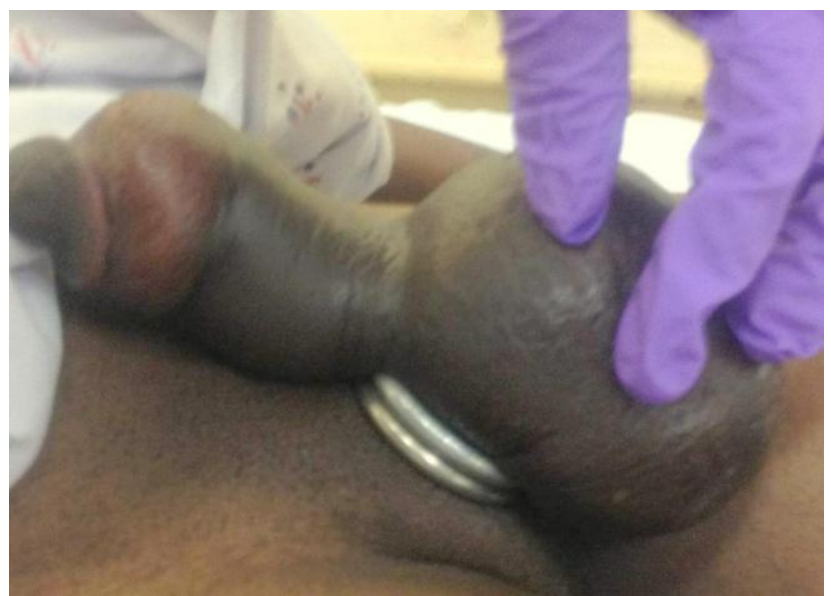

Figure 2: Lateral view demonstrating extensive edema of penis and srcotum, and the absense of broken skin.

Resultantly, the patient was taken to the operating room (OR) and placed under monitored anesthesia care (MAC). Then, the high speed Midas Rex Legend pneumatic orthopedic drill with metal cutter attachment was used to excise each ring (Fig. 3). Each ring was approached separately at the 3 and 9 o'clock positions. Throughout the procedure, continuous flow sterile water was used to keep the metal cool. A tongue blade was used to shield the external genitalia during the procedure. The entire operating time was approximately 15 minutes. The penis and scrotal skin showed no signs of ulceration or necrosis, and the patient was discharged the same day in good condition. He was covered with perioperative antibiotics, nonnarcotic analgesia, and supportive ice pack.

Outpatient follow-up occurred one month later in clinic at which time the patient denied any problems with urination, skin changes, pain, or swelling. His genitourinary physical exam was within normal limits (he scored a 24 out of 25 on the International Index of Erectile Function Questionnaire). The patient had complete recovery with good cosmetic and functional outcome.

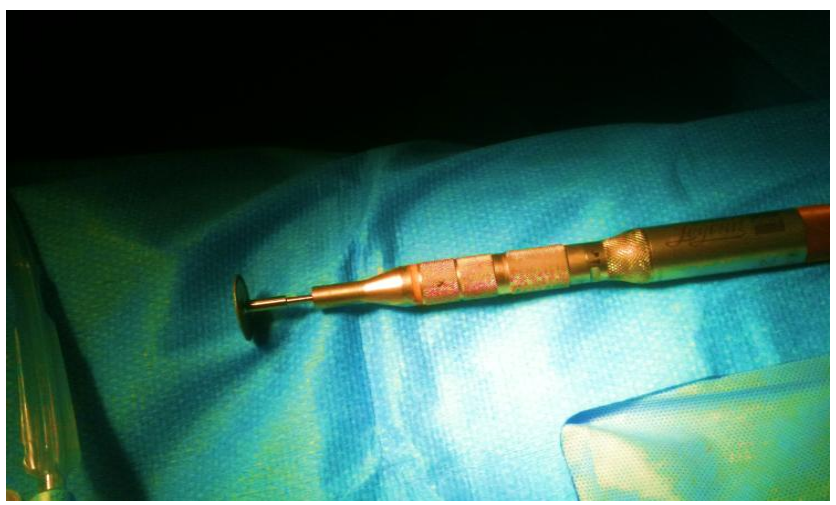

Figure 3: Midas Rex Legend pneumatic orthopedic drill with metal cutter attachment.

\subsection{Aim}

\section{DISCUSSION AND How-To-GUIDE}

We intend to describe a surgical technique used to remove penile constriction devices made of metal.

\subsection{Epidemiology}

Penile and scrotal strangulation occur uncommonly with sixty cases reported in the literature (1)(2). Most of the incidences are a result of a patient wanting to enhance his sexual pleasure and prolong his erection during intercourse or masturbation (5)(6).

\subsection{Anatomy}

In this case, the penis and scrotum were incarcerated at the base of the organs. A cock ring is a constrictive device that can be placed around the base of the penis and/or scrotum to slow the flow of blood in an erect penis thus prolonging the erection. Rings can be made of various materials including leather, rubber, silicone, nylon, or metal and are removable by device clamp. 


\subsection{Indications}

Upon arrival to the emergency department or clinic, the physician should attempt to remove the rings manually (3). The longer the genitals have been incarcerated, the more swelling and edema there will be, and the more likely it is that there will be injury to the skin, blood vessels, and lymph vessels leading to strangulation (3)(6).

\subsection{Preparation}

The patient should be given an explanation of the procedure making sure that he understands and is comfortable (7).

3.5.1 Sedation and pain control: Topical anesthesia can be used for penile and scrotal urological procedures to decrease pain. They are effective only after thirty minutes of application, and if the skin is intact, numbing the area might not be necessary (7). Sedation with monitored anesthesia care or general anesthesia is warranted to decrease the patient's anxiety and to decrease the risk of unintentional movements and potential injury (3)(7).

3.5.2 Equipment: The following materials should be assembled

- Midas Rex pneumatic orthopedic drill

- Tongue blade

- Emesis basin

- Sterile water

\subsection{Technique}

The management of penile and or scrotal strangulation injuries is based on observational studies described in case reports. The intervention will depend largely on the location of the constriction device and the genital area involved. The following technique works for metal cock rings:

- Sedate the patient with monitored anesthesia care (MAC) or general anesthesia.

- Place a tongue blade underneath the ring to displace it and protect the patient's skin.

- Use continuous flow of sterile water to keep the metal cool while drilling and to prevent thermal burns.

- Use the Midas Rex pneumatic orthopedic drill to cut through the rings on one side and then 180 degrees from there.

- Allow the rings to fall off into an emesis basin and dispose.

\subsection{Aftercare}

The genital tissue may be swollen and bruised for a few days after an injury. The patient should be advised not to use cock rings in the future and to refrain from sexual activity until swelling resolves. Follow-up should occur within 4 weeks for a genitourinary physical examination. The genitals should be carefully inspected for potential skin loss (7) or other abnormalities, and urinary and erectile function should be assessed. Finally, the patient must be encouraged to seek medical attention if he experiences genital pain, swelling, or incarceration in the future.

\section{TEACHING POINTS}

- Clinicians should use the least painful/traumatic technique to remove a constriction device from the genitals as soon as possible after incarceration.

- Analgesia and anesthesia are essential in management of patients with genital strangulation.

- Selection of tools needed for removal of a constriction device depends on [1] the resourcefulness of the clinicians, [2] the equipment available, [3] the size and thickness of the constricting object, and [4] the severity of injury.

- Prolonged strangulation of the genitals can lead to penile lymphedema, voiding dysfunction, erectile dysfunction, skin ulceration, necrosis, urethral cutaneous fistula, and colonization of hypertrophic skin. 


\section{REFERENCES}

1) Detweiler, M. Penile incarceration with metal objects a review of procedure choice based on penile trauma grade. Scand J Urol Nephr 2001, 35, 212-217.

2) Katz, D, Chin, W, Appu, S, Harper, M, et al. Novel extraction technique to remove a penilconstriction device. J Sex Med (2012);9, 937-940.

3) Cassidy, D, \& Mador, D. Genital incarceration: an unusual case report. Canadian Urological Association Journal 2010;4(3), E76-E78.

4) Lopes, R. L., Lopes, S. I., \& Lopes, R. N. Chronic penile strangulation. International Braz J Urol 2003; 29(4), 327-329

5) Silberstein, J, Grabowski, J, Lakin, C., et al. Case reports: Penile constriction devices. J Sex Med 2008; 5(7), 1747-1757

6) De, U, Maitra, D, Pal, S, et al. Mason at a surgical mission. African Journal of Medicine and Surgery 2011, 1(2), 13-14.

7) Bothner, J. (2014). Management of zipper injuries. In A. M. Stack \& J. F. Wiley (Eds.) UpToDate. Retrieved from http://www.uptodate.com/contents/management-of-zipper-injuries (accessed 14 Feb 2014) 